\title{
Vitamin D receptor is expressed within human carotid plaques and correlates with pro-inflammatory M1 macrophages
}

Federico Carbone ${ }^{1}$, Nathalie Satta ${ }^{2}$, Fabienne Burger ${ }^{3}$, Aline Roth ${ }^{3}$, Sébastien Lenglet ${ }^{3}$, Sabrina Pagano ${ }^{2}$, Pierre Lescuyer ${ }^{2}$, Maria Bertolotto ${ }^{1}$, Giovanni Spinella ${ }^{4}$, Bianca Pane ${ }^{4}$, Domenico Palombo ${ }^{4}$, Aldo Pende ${ }^{1,5}$, Franco Dallegri ${ }^{1,5}$, François Mach $^{3}$, Nicolas Vuilleumier $^{2}$, Fabrizio Montecucco ${ }^{1,5}$ 


\section{Abbreviation list}

CV: Cardiovascular

VDR: Vitamin D receptor

CEA: Carotid endarterectomy

NASCET: North American Symptomatic Carotid Endarterectomy Trial

ECST: European Carotid Surgery Trial

ACST: Asymptomatic Carotid Surgery Trial

NYHA: New York Heart Association

MACE: Major Adverse Cardiovascular Event

CRP: C-Reactive Protein

MACE: Major Acute Cardiovascular Event

CCL: chemokine (CC motif) ligand

INF: Interferon

TLR: Toll-like Receptor

IQR: interquartile range

ROC: Receiver Operating Characteristic Non-contrast Cranial Computed Tomography

NIHSS: National Institutes of Health Stroke Scale

AUC: Area under the curve

CI: Confidence Interval

HR: Hazard ratio

SD: Standard Deviation 


\section{Abstract}

The role of Vitamin D system in cardiovascular diseases remains controversial. Here, we investigated whether intraplaque levels of vitamin D receptor (VDR) predicted major adverse cardiovascular events (MACEs) at 18 month-follow-up and correlated with macrophage subsets in 164 patients undergoing endarterectomy for carotid stenosis. In human carotid plaque portions upstream and downstream the blood flow, VDR, lipid, collagen, as well as macrophage subsets were determined. Human primary monocytes were then differentiated in vitro to M1 and M2 macrophages and treated with $1,25(\mathrm{OH})_{2} \mathrm{D}_{3}$.

Intraplaque VDR positively correlated with total and M1 macrophages. According to the result of ROC curve analysis, downstream portions of plaques having high VDR expression were characterized by increased M1 macrophages. Kaplan-Meier analysis showed that the risk of MACEs was greater in patients having low downstream VDR levels $(8.2 \%$ vs. $1.3 \%$; $p=0.005)$. Cox proportional hazard regression analyses confirmed that MACE risk decreased with increasing downstream VDR (adjusted HR 0.78 [95\% CI 0.62-0.98]; $p=0.032$ ). In vitro, VDR expression was prevalent in M1, but not M2. Incubation of M1 macrophages with $1,25(\mathrm{OH})_{2} \mathrm{D}_{3}$, increased VDR expression and suppressed toll-like receptor 4 expression. These results suggest that low intraplaque VDR expression predict MACEs in patients with carotid stenosis potentially involving M1 macrophages.

Key words: Atherosclerosis, macrophage, major adverse cardiovascular events, vitamin D, vitamin D receptor. 


\section{Introduction}

Vitamin D deficiency was initially supposed to be a condition increasing cardiovascular (CV) risk [1], because low circulating levels of $25(\mathrm{OH}) \mathrm{D}_{3}$ (also known as vitamin $\mathrm{D}$ ) have been associated with classical CV risk factors, including hypertension, dyslipidaemia, insulin resistance, and overweight/obesity [2-5]. However, in subsequent randomized clinical trials, vitamin D supplementation strategies failed to improve CV outcome [1]. Poor stratification for confounding factors, large variability of compounds used for supplementation and the lack of consensus definition of vitamin D deficiency may partially explain these discouraging clinical results.

Furthermore, from a pathophysiological point of view, many studies support an important role of the vitamin D in the regulation of critical pathways involved in vascular physiology, such as the renin-angiotensin-aldosterone system [6], and fibroblast growth factor $23 /$ klotho axis [7] and the major influence of autocrine/paracrine-related properties of vitamin D. The discovery of extra-renal expression of CYP27B1 (the rate-limiting enzyme catalysing hydroxylation of $25(\mathrm{OH}) \mathrm{D}_{3}$ to the active form $1,25(\mathrm{OH})_{2} \mathrm{D}_{3}$ and the ubiquitous distribution of vitamin D receptor (VDR) further reinforced the importance of a local control of vitamin D signalling in modulating vascular pathologies. Several cell types involved in atherogenesis (i.e., vascular smooth muscle cells, endothelial cells, macrophages, and dendritic cells) showed a CYP27B1 activity and a VDR expression in vitro, highlighting the importance of an autocrine/paracrine control of vitamin D signalling [1]. However, these local effects of vitamin D were marginally investigated within human atherosclerotic plaques and resident inflammatory cells, such as macrophages. Furthermore, the potential role of local vitamin D on toll-like receptor expression (known to be relevant in atherogenesis) [8] on different macrophage subsets remains elusive. 
In this study, we assessed the prognostic value of the intraplaque VDR expression in upstream and downstream portions of carotid atherosclerotic plaques. In addition, we investigated the potential association between levels of VDR and the different subtype of macrophages. Finally, both function and membrane expression of VDR were assessed in vitro on different macrophage subsets (i.e. M1 and M2).

\section{Methods}

\section{$2.1 \quad$ Patients and clinical assessment}

From March 2008 to June 2011, we consecutively enrolled 201 patients with extra cranial high-grade internal carotid stenosis ( $>70 \%$ luminal narrowing) [9] in an observational cohort at a single center (IRCCS Azienda Ospedaliera Universitaria San Martino-IST Istituto Nazionale per la Ricerca sul Cancro, Genoa, Italy). Some of the cohort samples have already been used for analysis and published [10]. Among the total cohort ( $\mathrm{n}=201), 37$ carotid endarterectomy were missing, leaving 164 samples available for analyses that were included in the present sub-study. As previously described [11], all patients underwent elective carotid endarterectomy (CEA) according to the recommendations published by the North American Symptomatic Carotid Endarterectomy Trial (NASCET) [12], the European Carotid Surgery Trial (ECST) [13], and the Asymptomatic Carotid Surgery trial (ACST) [14]. The Ethics Committee of IRCCS Azienda Ospedaliera Universitaria San Martino-IST Istituto Nazionale per la Ricerca sul Cancro in Genoa, Italy approved this protocol, performed in accordance to the guidelines of the Declaration of Helsinki. Patients gave informed consent before entering in the study. The day prior to CEA, serum samples were obtained to measure circulating markers of cardiovascular vulnerability. Medications reported in Table 1 were not modified in the 2 months prior to enrolment. Exclusion criteria were: spontaneous cerebral embolism up to 30 minutes preoperatively or during the dissection phase of the operation, malignant 
hypertension, acute coronary artery disease, any cardiac arrhythmias, congestive heart failure (II, III, and IV New York Heart Association [NYHA] classes), liver or renal disorder or function abnormalities, acute and chronic infectious diseases, autoimmune and rheumatic diseases, cancer, endocrine diseases, inflammatory bowel diseases and anti-inflammatory (other than aspirin) medications, oral anticoagulant treatments, and hormone, cytokine or growth factor therapies.

\subsection{Study endpoints}

We performed a pilot study to investigate human intraplaque VDR expression levels. The primary end-point of the study was to determine if VDR intraplaque expression levels at time of CEA could predict the occurrence of major adverse cardiovascular events (MACEs, defined as fatal or non-fatal acute coronary syndrome and stroke) over a follow-up of 18 months [10]. The secondary end-points consisting in determining the potential correlations between circulating vitamin D levels or intraplaque VDR with histological parameters of inflammation in upstream and downstream portions of carotid plaques. Two independent investigators who were blinded to the biochemical and histological analyses adjudicated the study endpoints. Information was obtained during a check-up visit at 18 months and was further confirmed by checking patients' medical file, targeting medical history relevant to the study endpoint, as noted in their medical file.

\subsection{Detection of biochemical and inflammatory biomarkers}

Routine auto-analysers were used to assay haematological parameters and blood chemistry including total cholesterol, low- and high-density lipoprotein cholesterol, triglycerides fibrinogen and glycaemia. Total $25(\mathrm{OH})$ vitamin $\mathrm{D}_{3}$ measurements were performed at the Division of Laboratory Medicine of the Geneva University Hospitals on a Cobas 6000 
immunoanalyzer (Roche Diagnostics, Rotkreuz, Switzerland) using Elecsys Vitamin D Total reagents (Roche Diagnostics). Inter-assay coefficients of variation $(n=68)$ of the method were $8.0 \%$ at $47.9 \mathrm{nmol} / 1$ and $6.3 \%$ at $108.7 \mathrm{nmol} / 1$. Serum levels of C-reactive protein (CRP) and the CC chemokines CCL2, CCL3, CCL4, CCL5 (all from R\&D systems, Minneapolis, MN) were measured by colorimetric enzyme-linked immunosorbent assay (ELISA) following the manufacturer's instructions. The limits of detection were $31.25 \mathrm{pg} / \mathrm{ml}$ for CRP, $15.60 \mathrm{pg} / \mathrm{ml}$ for CCL2, $7.81 \mathrm{pg} / \mathrm{ml}$ for CCL3, $15.60 \mathrm{pg} / \mathrm{ml}$ for CCL4, $15.60 \mathrm{pg} / \mathrm{ml}$ for CCL5. Mean intraand inter-assay coefficients of variation were $<8 \%$ for all markers measured by ELISA.

\subsection{Endarterectomy specimen processing}

As previously reported [11], the internal carotid plaque specimens were cut right after surgical excision, perpendicularly to the long axis through the point of maximum stenosis to obtain two portions (upstream and downstream of the blood flow). Each portion was further divided perpendicularly to the long axis in the middle into two sub-segments. Upstream and downstream carotid specimens were then frozen in cryoembedding medium for histological analysis and serially cut in $7-\mu \mathrm{m}$ sections.

\subsection{Oil Red O staining for lipid content}

Eight sections per each portion (upstream and downstream the blood flow) separated by 105 $\mu \mathrm{m}$ from each other were stained and counterstained with Mayer hemalune's solution, as previously described $[11,15]$. Quantifications were performed with MetaMorph ${ }^{\mathrm{TM}} 6$ software. $^{\mathrm{m}}$ Data were calculated as ratios of stained area on total lesion area.

\subsection{Sirius red staining for collagen content}


Eight section per each portion (upstream and downstream the blood flow) separated by 105 $\mu \mathrm{m}$ from each other were stained as previously described $[11,15]$. The sections were photographed under light microscopy (in order to evaluate total collagen content) and under polarization microscopy. Total collagen content was quantified by MetaMorph ${ }^{\mathrm{TM}} 6$ software. $^{\circ}$ Results on total collagen were presented as percentages of stained area on total lesion area.

\subsection{Immmunostaining of endarterectomy specimens}

Eight sections per each portion separated by $105 \mu \mathrm{m}$ from each other were fixed in acetone at room temperature and immunostained with specific antibodies. For detecting VDR, we used anti-human VDR (dilution: 1:500; Abcam, UK). For detecting macrophages, we used antihuman CD68 (markers of total macrophages, diluted: 1:100; Dako Corporation, CA), antihuman CD86 (marker of M1 macrophages, diluted: 1:100; GeneTex Inc., Irvine, CA), antihuman HLA-DR (marker of M1 macrophages, diluted 1:100; Dako Corporation), and antihuman CD163 (marker of M2 macrophages, diluted: 1:50; AbD Serotec, Oxford, UK) [16]. Quantifications were performed using MetaMorph ${ }^{\mathrm{TM}} 6$ software. Data were presented as percentages of stained area on total lesion area.

\subsection{Monocyte purification}

Monocytes were isolated from blood buffy coats of healthy volunteers provided by the Geneva Hospital blood transfusion center, as previously described [17]. Monocyte purity was routinely controlled by flow cytometry and the isolated cell population consisted of CD14 $4^{+}$

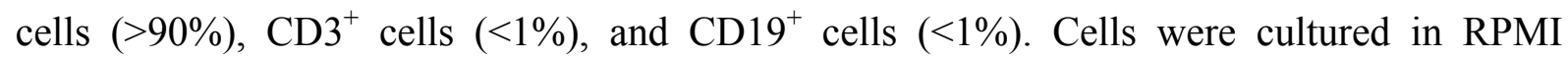
containing 10\% FBS (Gibco BRL-Life Technologies, Grand Island, NY) [18].

\subsection{Mono-macrophage polarization and treatment}


Mono-macrophages were respectively polarized toward M1 or M2 subsets either by $24 \mathrm{~h}$ incubation with $500 \mathrm{U} / \mathrm{ml}$ of interferon(INF)-gamma (Roche Diagnostic, Mannheim, Germany) or $20 \mathrm{ng} / \mathrm{ml}$ of interleukin (IL)-4 (PeproTech, Inc, London, UK) (16). When indicated, $100 \mathrm{nmol} / 1$ of vitamin $1,25(\mathrm{OH})_{2} \mathrm{D}_{3}$ (Sigma-Aldrich Chemie GmbH, Germany) were added in the medium for $24 \mathrm{~h}$ or $48 \mathrm{~h}$ [19].

\subsection{Flow Cytometry analysis}

VDR and toll-like receptor (TLR)-4 expression were determined on non-polarized (M0) or polarized (M1 or M2) macrophages after treatment with INF-gamma or IL-4 for 24h (day 1). In addition, VDR and TLR4 expression were determined after additional treatment with 100 $\mathrm{nmol} / 1 \mathrm{1}, 25(\mathrm{OH})_{2} \mathrm{D}_{3}$ for $24 \mathrm{~h}$ or $48 \mathrm{~h}$ (day 2 and day 3 , respectively). For VDR staining, $5 \times 10^{5}$ cells per condition were fixed with $4 \%$ of PAF for $10 \mathrm{~min}$, then permeabilized with $0.1 \%$ saponin in PBS-2\% BSA and incubated with mouse monoclonal anti-CD32 and anti-CD64 antibodies $\left(0.5 \mu \mathrm{g} / 5 \times 10^{5}\right.$ cells) (Biolegend) for $10 \mathrm{~min}$. Subsequently, of $0.5 \mu \mathrm{g}$ of rabbit polyclonal anti-human VDR (ab3508, Abcam) were added for 30 min. After washing, cells were incubated with $4 \mu \mathrm{g} / \mathrm{ml}$ of AF488-conjugated goat anti-rabbit polyclonal antibodies (Invitrogen Co., Carlsbad, CA) for $30 \mathrm{~min}$. For TLR4 staining, $5 \times 10^{5}$ cells per condition were incubated with the mix of mouse monoclonal anti-CD32 and anti-CD64 antibodies in PBS2\% BSA for $10 \mathrm{~min}$ on ice, after which $8 \mu \mathrm{g} / \mathrm{ml}$ of APC-mouse monoclonal anti-TLR4 antibodies (HTA125, Biolegend, San Diego, CA) were added for $30 \mathrm{~min}$. Staining was quantified with ACCURI C6 flow cytometer (BD Biosciences, San Jose, CA) and analysed using FlowJo data analysis software (version 10.0.7) (Flow Jo LLC, Ashland, OR).

\subsection{Statistical analysis}


Analyses were performed with IBM SPSS Statistics for Windows, Version 21.0 (IBM CO., Armonk, NY), and STATISTICA 12 (StatSoft, Inc. Tusla, OK). In the clinical study, categorical data are presented as relative and absolute frequencies, whereas continuous variables were expressed median and interquartile range (IQR). Intergroup comparisons were drawn by Fisher's exact test and Mann-Whitney $U$-test, as appropriate. Ranked Spearman correlation coefficients were performed to establish correlations between serum and intraplaque biomarkers in both upstream and downstream portions of atherosclerotic plaque. The prognostic ability of VDR was assessed in a post-hoc manner based upon receiver operator characteristic (ROC) curve, using MedCalc 12.5 (MedCalc Software, Ostend, Belgium). The area under the curve (AUC) was given with 95\% confidence interval (CI) and the cut-off point of VDR was calculated maximizing the sensitivity in accordance to the Youden's index. Kaplan-Meier survival analysis with Log Rank test was performed to estimate cumulative event rate during 18 months after CEA and to calculate the corresponding risk difference according to categorized VDR. Finally, the effect of VDR expression on MACE risk was estimated by Cox proportional hazards models and expressed with hazard ratios (HR) and 95\% CI. In the multivariate model, we adjusted for age, gender and serum levels of $25(\mathrm{OH}) \mathrm{D}_{3}$. In the basic research study, the data were presented as mean \pm standard deviation (SD). Statistical analyses were performed using Mann-Whitney U-test. For all statistical analyses a 2 -sided $p$-value $<0.05$ was considered as statistically significant.

\section{Results}

\subsection{Patient characteristics}

Clinical and biochemical characteristics of the study cohort, including medications, are listed in Table 1. Median patient age was $72(67-77)$ years, with a high prevalence of males $(66.5 \%)$ and hypertensive patients $(71.9 \%)$. Biochemical parameters, haematological are shown in 
Table 1, whereas circulating levels of vitamin $25(\mathrm{OH}) \mathrm{D}_{3}, \mathrm{CRP}$ and $\mathrm{CC}$ chemokines are reported in Table 2. These results indicate that the cohort of patients was not affected by relevant acute inflammation.

\subsection{Circulating vitamin $25(\mathrm{OH}) \mathrm{D}_{3}$ and intraplaque VDR levels correlate with intraplaque inflammatory parameters}

A significant correlation between serum levels of vitamin $25(\mathrm{OH}) \mathrm{D}_{3}$ and intraplaque lipid content $(r=0.242 ; p=0.003)$ was observed in the upstream portions of the plaques (Table 3$)$. With regard to the downstream portion, serum vitamin $25(\mathrm{OH}) \mathrm{D}_{3}$ showed a positive correlation with VDR $(r=0.166 ; p=0.038)$ and total macrophages $(r=0.177 ; p=0.027)$ (Table 3). As reported in Table 4, we also analysed the relationship between intraplaque VDR levels and different intraplaque parameters of inflammation. In the upstream portions, VDR directly correlated with the M1 macrophage markers CD86 $(r=0.371 ; p<0.001)$ and HLA-DR $(r=0.402 ; p<0.001)$ (Table 4 and Figure 1). Similarly, in the downstream portions, VDR directly correlated with total macrophages $(r=0.365 ; p<0.001)$ and the M1 markers CD86 $(r=0.279 ; p<0.001)$ and $\underline{\text { HLA-DR }}(r=0.304 ; p<0.001)$ (Table 4 and Figure 2$)$. In both upstream and downstream portions of carotid plaques, positive areas for VDR corresponded to intraplaque zones positive for M1 macrophages markers (Figures 1 and 2). Conversely, no significant correlation was found between VDR and lipids, collagen and M2 macrophages $\left(\mathrm{CD} 163^{+}\right.$cells) in both upstream and downstream portion of the plaque (Figures 1 and 2).

\subsection{VDR levels in downstream portion of carotid plaques predict future MACES}

ROC curve analysis showed that upstream expression of VDR failed to predict the occurrence of MACEs (Figure 3A). Conversely, downstream expression of VDR had significant prognostic accuracy to predict subsequent MACEs (AUC 0.718 [95\% CI 0.642-0.785]; 
$p=0.001$ ) (Figure 3B). In accordance with the Youden Index, a downstream VDR expression $7.13 \%$ was identified as the best cut-off point, having a sensitivity of $100 \%$ and a specificity of $49.03 \%$. The downstream plaques were then categorized by low $(\leq 7.13 \%)$ or high $(>7.13 \%)$ VDR levels. At this cut-off, Kaplan-Meier analysis indicated that patients with low VDR expression had a higher rate of MACEs than patients expressing high VDR levels (Figure 4). Of the 9 MACEs observed during 18 months of follow-up, 8 occurred in the group with low VDR expression, whereas only 1 was observed in patients with VDR values above the cut-off $(8.2 \%$ vs. $1.3 \%$; $<0.001)$ (Figure 4$)$. Cox proportional hazard regression analyses further confirmed these findings. The risk of MACEs decreased with increasing VDR expression in the downstream portion of the plaque (HR 0.82 [95\% CI 0.67-0.99]; $\mathrm{p}=0.046$ ) (Table 5). These results remained statistically significant also after adjustment for age, gender, and serum levels of vitamin $25(\mathrm{OH}) \mathrm{D}_{3}(\mathrm{HR} 0.78$ [95\% CI $0.62-0.98] ; p=0.032$ ) (Table 5).

\subsection{VDR levels in downstream portions of carotid plaques are associated with intraplaque content of M1 macrophages}

The amount of different macrophages subsets was quantified according to low versus high VDR expression in downstream regions of plaques. In the upstream portions, intraplaque content of macrophages did not differ between groups, even when analysing the M1 and M2 subsets separately (Figure 5A-D). Conversely, downstream portions with high VDR levels were characterized by an increased M1 macrophage content $(p=0.001)$ (Figure 5A). Specifically, high M1 macrophages were found in plaques with high VDR levels, as indicated by the high expression of CD86 $(p=0.002)$ and $\underline{\text { HLA-DR }}(p=0.010)$ (Figure 5B and C). No difference for M2 macrophages were observed (Figure 5D). 
3.5 VDR is highly expressed on M1 macrophages and modulates the surface expression of toll-like receptor 4

To further investigate the expression and activity of VDR on human macrophage subsets, we performed an in vitro analysis of VDR expression on the M1 and M2 macrophage subtype after differentiation. As compared to undifferentiated macrophages (M0), M1 (but not M2) macrophages showed a significant and persistent VDR expression at 24, 48 and 72 hours (Figure 6A). Incubation with $1,25(\mathrm{OH})_{2} \mathrm{D}_{3}$ further up-regulated VDR expression on $\mathrm{M} 1$ macrophages (Figure 6B). We also observed that M1 macrophages expressed high levels of TLR4, as compared with M2 (Figure 6C). Incubation with vitamin $1,25(\mathrm{OH})_{2} \mathrm{D}_{3}$ abrogated TLR4 expression on M1 macrophages, with a significant effect observed at 48 hours (Figure 6D).

\section{Discussion}

The novel finding of this study resides in the fact that circulating levels of vitamin D were not predictive of subsequent MACE, whereas low VDR expression within CEA biopsies was. These results extend previous negative finding regarding the possible CV prognostic value of circulating vitamin D levels and further emphasizes the importance of local vitamin D expression in atherogenesis and its related complications in humans. These results are in accordance with previous in vivo results derived from monkey's models, which demonstrated an inverse relationship between VDR and atherosclerotic the plaque size [20]. However, the authors did not clarify which cells expressed VDR and whether low VDR expression was a result of plaque progression and if it contributed directly to atherosclerotic plaque vulnerability. Furthermore, in this study we analysed separately upstream and downstream portions of carotid plaques allowing us to evaluate the effect of shear stress on intraplaque macrophages polarization. We retrieved a positive correlation between intraplaque VDR and 
total macrophages in the downstream portions of the plaques. In addition, we observed that intraplaque VDR was positively correlated with CD86 and HLA-DR, two hallmarks of M1 macrophages [16]. Despite highly speculative, we believe that both different hemodynamic $\underline{\text { forces and cell recruitment/activation might influence expression of VDR as well as other }}$ receptors on M1 macrophages in human carotid plaques [21, 22]. However, since M1 macrophages are traditionally considered as a pro-inflammatory subset associated with plaque vulnerability [16], this association with VDR expression is rather intriguing as the opposite observation would have been expected. Indeed the activation of vitamin D signalling was shown to reverse pro-atherogenic cholesterol metabolism in macrophages by downregulating the expression of scavenger receptors (SR-A1 and CD36), TLRs [23, 24] and promoting cholesterol efflux [25-27]. Further contrasting with current results, vitamin $1,25(\mathrm{OH}) 2 \mathrm{D}_{3}$ was shown to promote the M1 switching to the anti-inflammatory M2 phenotype $[19,28]$. Nevertheless, the fact that vitamin D up-regulated VDR expression on M1 macrophages could be interpreted as a negative feedback loop mechanism to contain the pro-inflammatory M1 response. The fact that vitamin D treatment decreased TLR4 expression on M1 macrophages, and that low VDR expression in the downstream part of atherosclerotic plaque acted as an independent predictor of MACE during follow-up is in line with this hypothesis. These results suggested an autocrine control of vitamin D with potential role as feedback mechanism with inhibitory effect on macrophage activity $[29,30]$, through the modulation of TLR4 expression. In this context, the down-regulation of TLR4 might represent a mechanism of switching from M1 to M2 macrophages as previously suggested [31], but this intriguing hypothesis requires further investigations. In addition, whether this autocrine control of VDR could also modulate different Vitamin D enzymes, including the hydroxylases CYP27A1, CYP27B1, and CYP24A [1], remains unknown. 
This study has many limitations. As first, the lack of a full description of the intraplaque vitamin D system (including enzymes) that should be addressed in future researches. Secondly, knowing whether portion of atherosclerotic plaque with low VDR had a higher TL4 expression would have reinforced the present in vitro results. Nevertheless, due to the limited amount of available material, we could not assed TLR expression in human atherosclerotic plaques. Thirdly, the relative small sample size and the involvement of a single center for patient recruitment may represent a further limitation in term of possible selection bias. Finally, the post-hoc definition of the VDR cut-off point by ROC curve prompt us to prospectively validate the defined cut-off in another larger prospective cohort.

In conclusion, the present study demonstrated that low VDR levels in downstream portions of carotid plaques predicted MACEs, but not the plasmatic levels of vitamin D. In addition, intraplaque VDR levels were positively associated with M1 macrophage content. In vitro, incubation with $1,25(\mathrm{OH})_{2} \mathrm{D}_{3}$ on differentiated $\mathrm{M} 1$ macrophages increased VDR and decreased TLR4 expression on cell surface membranes, suggesting an anti-inflammatory effect of Vitamin D on this cell subset. Although not conclusive, our results strongly indicate VDR as an active anti-inflammatory mediator within atherosclerotic plaques.

\section{Acknowledgments}

This study was supported by a grant from the European Commission (FP7-INNOVATION I HEALTH-F2-2013-602114; Athero-B-Cell: Targeting and exploiting B cell function for treatment in cardiovascular disease) and a grant from the Swiss National Science Foundation

Grant to Dr. F. Montecucco (\#310030_152639/1) and to Dr N. Vuilleumier (\#310030_140736). This study was supported by grants from the Foundation "Gustave and Simone Prévot" and the F4LabMed to Dr. F. Carbone. 


\section{References}

[1] Carbone F, Montecucco F. The role of the intraplaque vitamin $d$ system in atherogenesis. Scientifica (Cairo) 2013;2013:620504.

[2] Carbone F, Mach F, Vuilleumier N, Montecucco F. Potential pathophysiological role for the vitamin D deficiency in essential hypertension. World J Cardiol 2014;6:260276.

[3] Zittermann A, Gummert JF, Borgermann J. The role of vitamin D in dyslipidemia and cardiovascular disease. Curr Pharm Des 2011;17:933-942.

[4] Al-Shoumer KA, Al-Essa TM. Is there a relationship between vitamin D with insulin resistance and diabetes mellitus? World J Diabetes 2015;6:1057-1064.

[5] Strange RC, Shipman KE, Ramachandran S. Metabolic syndrome: A review of the role of vitamin $\mathrm{D}$ in mediating susceptibility and outcome. World $\mathrm{J}$ Diabetes 2015;6:896-911.

[6] Vaidya A, Brown JM, Williams JS. The renin-angiotensin-aldosterone system and calcium-regulatory hormones. J Hum Hypertens 2015;29:515-521.

[7] Quarles LD. Skeletal secretion of FGF-23 regulates phosphate and vitamin D metabolism. Nat Rev Endocrinol 2012;8:276-286.

[8] Montecucco F, Braunersreuther V, Burger F, Lenglet S, Pelli G, Carbone F, et al. Anti-apoA-1 auto-antibodies increase mouse atherosclerotic plaque vulnerability, myocardial necrosis and mortality triggering TLR2 and TLR4. Thromb Haemost $2015 ; 114: 410-422$.

[9] North American Symptomatic Carotid Endarterectomy Trial C. Beneficial effect of carotid endarterectomy in symptomatic patients with high-grade carotid stenosis. $\mathrm{N}$ Engl J Med 1991;325:445-453. 
[10] Vuilleumier N, Montecucco F, Spinella G, Pagano S, Bertolotto M, Pane B, et al. Serum levels of anti-apolipoprotein A-1 auto-antibodies and myeloperoxidase as predictors of major adverse cardiovascular events after carotid endarterectomy. Thromb Haemost 2013;109:706-715.

[11] Montecucco F, Lenglet S, Gayet-Ageron A, Bertolotto M, Pelli G, Palombo D, et al. Systemic and intraplaque mediators of inflammation are increased in patients symptomatic for ischemic stroke. Stroke 2010;41:1394-1404.

[12] Barnett HJ, Taylor DW, Eliasziw M, Fox AJ, Ferguson GG, Haynes RB, et al. Benefit of carotid endarterectomy in patients with symptomatic moderate or severe stenosis. North American Symptomatic Carotid Endarterectomy Trial Collaborators. N Engl J Med 1998;339:1415-1425.

[13] Randomised trial of endarterectomy for recently symptomatic carotid stenosis: final results of the MRC European Carotid Surgery Trial (ECST). Lancet 1998;351:13791387.

[14] Halliday A, Mansfield A, Marro J, Peto C, Peto R, Potter J, et al. Prevention of disabling and fatal strokes by successful carotid endarterectomy in patients without recent neurological symptoms: randomised controlled trial. Lancet 2004;363:14911502.

[15] Montecucco F, Di Marzo V, da Silva RF, Vuilleumier N, Capettini L, Lenglet S, et al. The activation of the cannabinoid receptor type 2 reduces neutrophilic proteasemediated vulnerability in atherosclerotic plaques. Eur Heart J 2012;33:846-856.

[16] Stoger JL, Gijbels MJ, van der Velden S, Manca M, van der Loos CM, Biessen EA, et al. Distribution of macrophage polarization markers in human atherosclerosis. Atherosclerosis 2012;225:461-468. 
[17] Montecucco F, Vuilleumier N, Pagano S, Lenglet S, Bertolotto M, Braunersreuther V, et al. Anti-Apolipoprotein A-1 auto-antibodies are active mediators of atherosclerotic plaque vulnerability. Eur Heart J 2011;32:412-421.

[18] Satta N, Kruithof EK, Fickentscher C, Dunoyer-Geindre S, Boehlen F, Reber G, et al. Toll-like receptor 2 mediates the activation of human monocytes and endothelial cells by antiphospholipid antibodies. Blood 2011;117:5523-5531.

[19] Zhang X, Zhou M, Guo Y, Song Z, Liu B. 1,25-Dihydroxyvitamin D(3) Promotes High Glucose-Induced M1 Macrophage Switching to M2 via the VDR-PPARgamma Signaling Pathway. Biomed Res Int 2015;2015:157834.

[20] Schnatz PF, Nudy M, O'Sullivan DM, Jiang X, Cline JM, Kaplan JR, et al. The quantification of vitamin D receptors in coronary arteries and their association with atherosclerosis. Maturitas 2012;73:143-147.

[21] Kwak BR, Back M, Bochaton-Piallat ML, Caligiuri G, Daemen MJ, Davies PF, et al. Biomechanical factors in atherosclerosis: mechanisms and clinical implications. Eur Heart J 2014;35:3013-3020, 3020a-3020d.

[22] Seneviratne AN, Cole JE, Goddard ME, Park I, Mohri Z, Sansom S, et al. Low shear stress induces M1 macrophage polarization in murine thin-cap atherosclerotic plaques. J Mol Cell Cardiol 2015;89:168-172.

[23] Ojaimi S, Skinner NA, Strauss BJ, Sundararajan V, Woolley I, Visvanathan K. Vitamin D deficiency impacts on expression of toll-like receptor-2 and cytokine profile: a pilot study. J Transl Med 2013;11:176.

[24] Verma R, Jung JH, Kim JY. 1,25-Dihydroxyvitamin D3 up-regulates TLR10 while down-regulating TLR2, 4, and 5 in human monocyte THP-1. J Steroid Biochem Mol Biol 2014;141:1-6. 
[25] Riek AE, Oh J, Bernal-Mizrachi C. 1,25(OH)2 vitamin D suppresses macrophage migration and reverses atherogenic cholesterol metabolism in type 2 diabetic patients. J Steroid Biochem Mol Biol 2013;136:309-312.

[26] Riek AE, Oh J, Darwech I, Moynihan CE, Bruchas RR, Bernal-Mizrachi C. 25(OH) vitamin D suppresses macrophage adhesion and migration by downregulation of ER stress and scavenger receptor A1 in type 2 diabetes. J Steroid Biochem Mol Biol 2014;144 Pt A:172-179.

[27] Karkeni E, Marcotorchino J, Tourniaire F, Astier J, Peiretti F, Darmon P, et al. Vitamin D limits chemokine expression in adipocytes and macrophage migration in vitro and in male mice. Endocrinology 2015;156:1782-1793.

[28] Riek AE, Oh J, Sprague JE, Timpson A, de las Fuentes L, Bernal-Mizrachi L, et al. Vitamin D suppression of endoplasmic reticulum stress promotes an antiatherogenic monocyte/macrophage phenotype in type 2 diabetic patients. $\mathrm{J}$ Biol Chem $2012 ; 287: 38482-38494$.

[29] Liu PT, Stenger S, Li H, Wenzel L, Tan BH, Krutzik SR, et al. Toll-like receptor triggering of a vitamin D-mediated human antimicrobial response. Science; 2006;24;311(5768):1770-3.

[30] Di Rosa M, Malaguarnera G, De Gregorio C, Palumbo M, Nunnari G, Malaguarnera L. Immuno-modulatory effects of vitamin D3 in human monocyte and macrophages. Cell Immunol 2012;280:36-43.

[31] Kawanishi N, Yano H, Yokogawa Y, Suzuki K. Exercise training inhibits inflammation in adipose tissue via both suppression of macrophage infiltration and acceleration of phenotypic switching from M1 to M2 macrophages in high-fat-dietinduced obese mice. Exerc Immunol Rev 2010;16:105-118. 


\section{Figure Legends}

Figure 1. Representative microphotographs of the upstream portion of human carotid atherosclerotic plaques. Immunostainings for total lipid content (Oil Red O), vitamin D receptor (VDR), total macrophage infiltrate $\left(\mathrm{M} \phi ; \mathrm{CD}^{+} 8^{+}\right.$cells), $\mathrm{M} 1$ macrophages $\left(\mathrm{CD} 86^{+}\right.$and $\underline{\text { HLA-DR }}^{+}$cells), and M2 macrophages $\left(\mathrm{CD} 163^{+}\right.$cells) were performed. The arrows and high magnifications pictures show that VDR and M1 macrophage subset localize in the same regions of atherosclerotic plaques.

Figure 2. Representative microphotographs of the downstream portions of carotid plaques. Immunostainings for total lipid content (Oil Red O), vitamin D receptor (VDR), total macrophage infiltrate $\left(\mathrm{M} \phi ; \mathrm{CD}^{+}\right.$cells $), \mathrm{M} 1$ macrophages $\left(\mathrm{CD} 86^{+}\right.$and $\underline{\text { HLA-DR }}{ }^{+}$ cells), and M2 macrophages $\left(\mathrm{CD}_{163}{ }^{+}\right.$cells) were performed. The arrows and high magnifications pictures show that VDR and M1 macrophage subset localized in the same regions of atherosclerotic plaques.

Figure 3. Receiver operator characteristic (ROC) curve analysis for VDR expression in upstream and downstream portions of carotid plaques. The predictive value of VDR expression toward the occurrence of major adverse cardiovascular events (MACEs) at 18month follow-up was tested. A. VDR expression in upstream portions. B. VDR expression in downstream portions.

Figure 4. Low downstream expression of VDR is associated with increased rate of major adverse cardiovascular events (MACEs) at 18 month-follow up. Kaplan-Meier curve according to low (VDR -: $\leq 7.13 \%$ ) and high (VDR +: $>7.13 \%$ ) VDR expression levels in downstream regions. 
Figure 5. Low expression of VDR in downstream portions of carotid plaques is associated with an increase in M1 macrophage content. Macrophage contant in both up and downstream portions of atherosclerotic plaques was analysed considering low (VDR -: $\leq 7.13 \%$ ) and high (VDR +: $>7.13 \%$ ) VDR expression levels in downstream regions. A. Total macrophage $(\mathrm{CD} 68+$ area) content. B. M1 macrophage $(\mathrm{CD} 86+$ area $)$ content. C. M1 macrophage ( $\underline{\text { HLA-DR }}+$ area) content. D. M2 macrophage (CD163+ area) content.

Figure 6. Treatment with vitamin $D$ in vitro upregulates VDR and downregulates TLR4 expression on M1 macrophages. VDR and TLR4 expression was assessed on M1 and M2 macrophages in vitro in the presence or absence of $100 \mathrm{nmol} / 1$ of $1,25(\mathrm{OH})_{2} \mathrm{D}_{3}$ (the active form of vitamin D). A. VDR expression on M1 and M2 macrophages up to 3 days of culture. B. VDR expression on $\mathrm{M} 1$ macrophages exposed to $1,25(\mathrm{OH})_{2} \mathrm{D}_{3}$ or control medium. $\mathbf{C}$. TLR4 expression on M1 and M2 macrophages up to 3 days of culture. D. TLR4 expression on M1 macrophages exposed to $1,25(\mathrm{OH})_{2} \mathrm{D}_{3}$

or control medium. 
Figure 1

\author{
Upstream
}
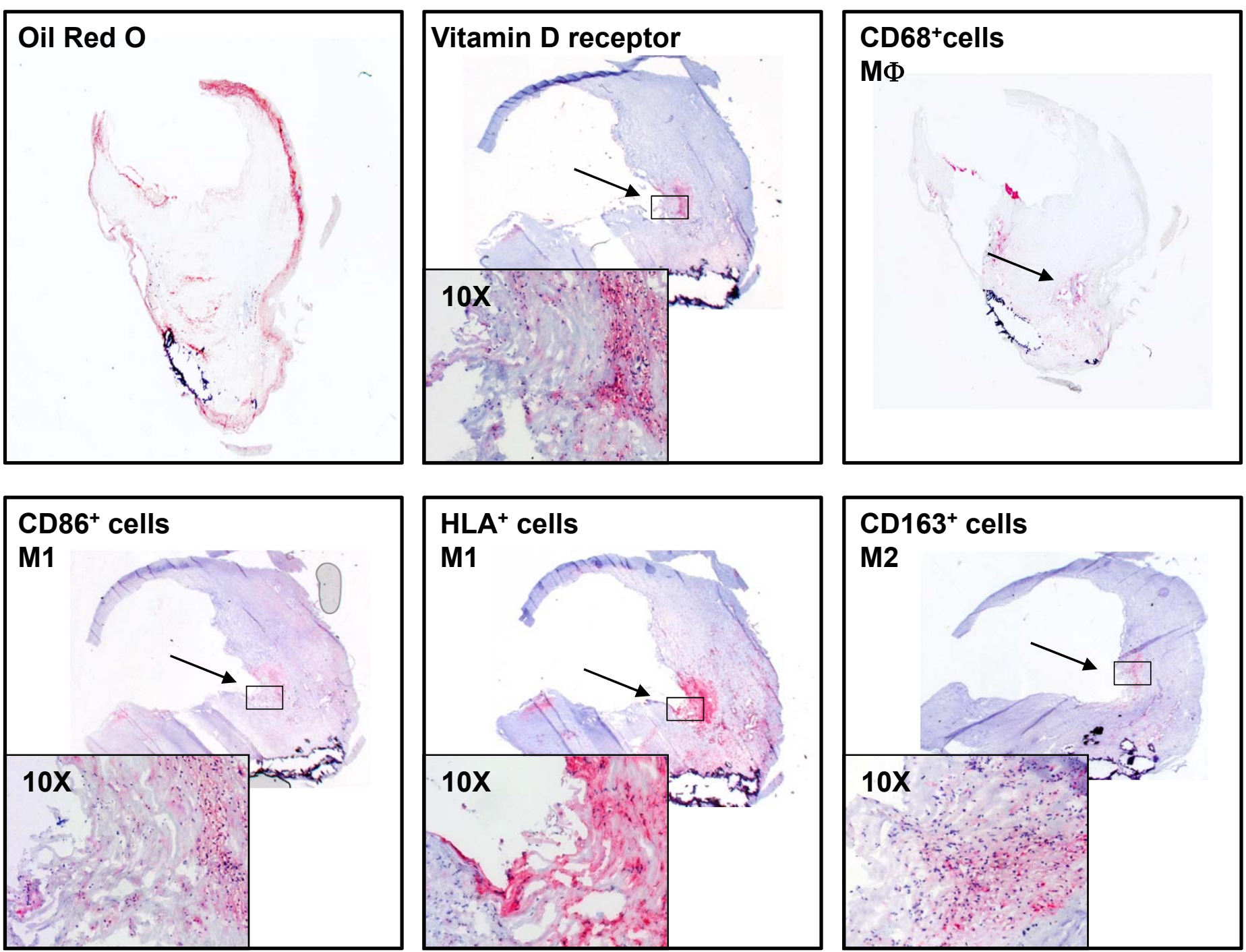
Figure 2

Downstream
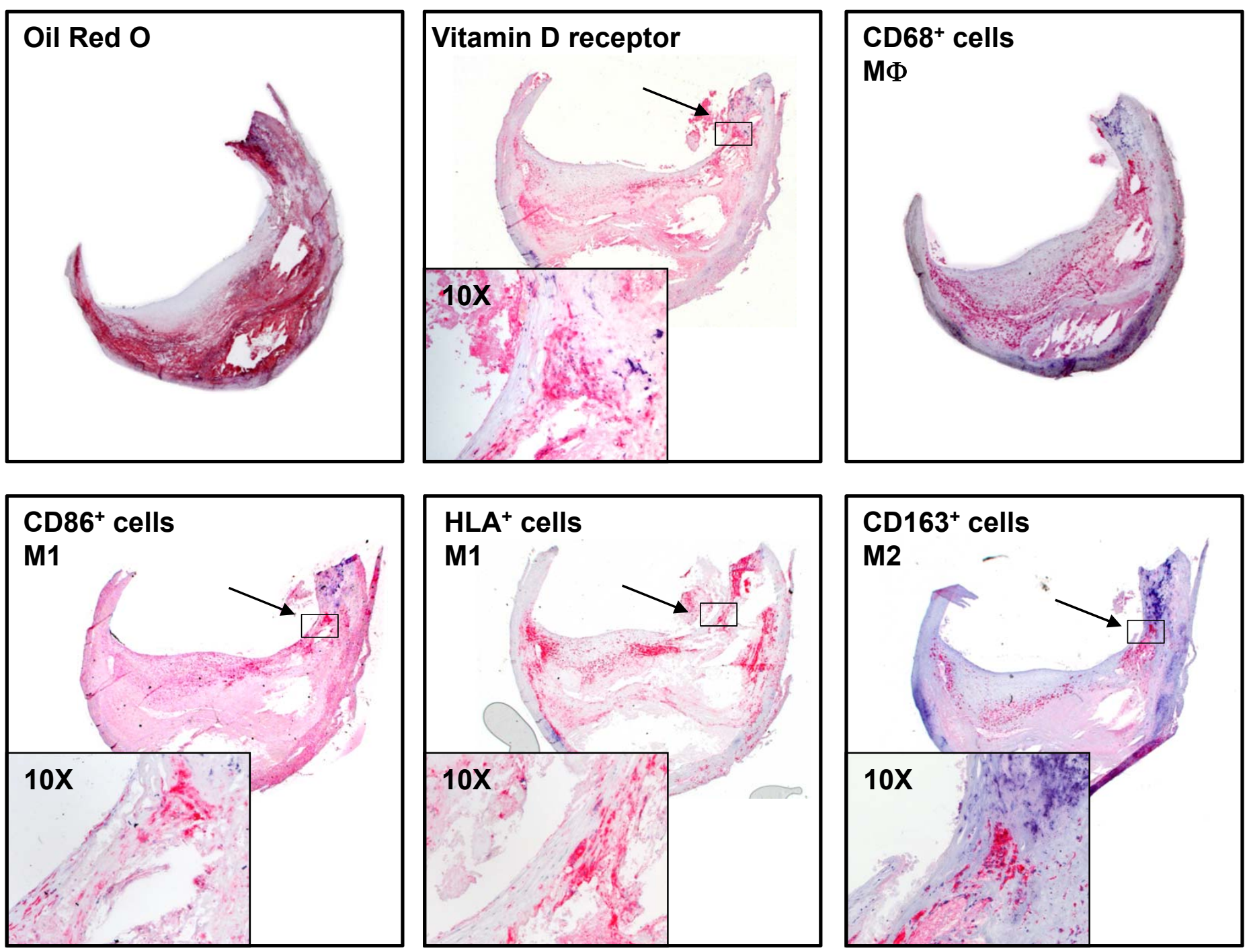
Figure 3
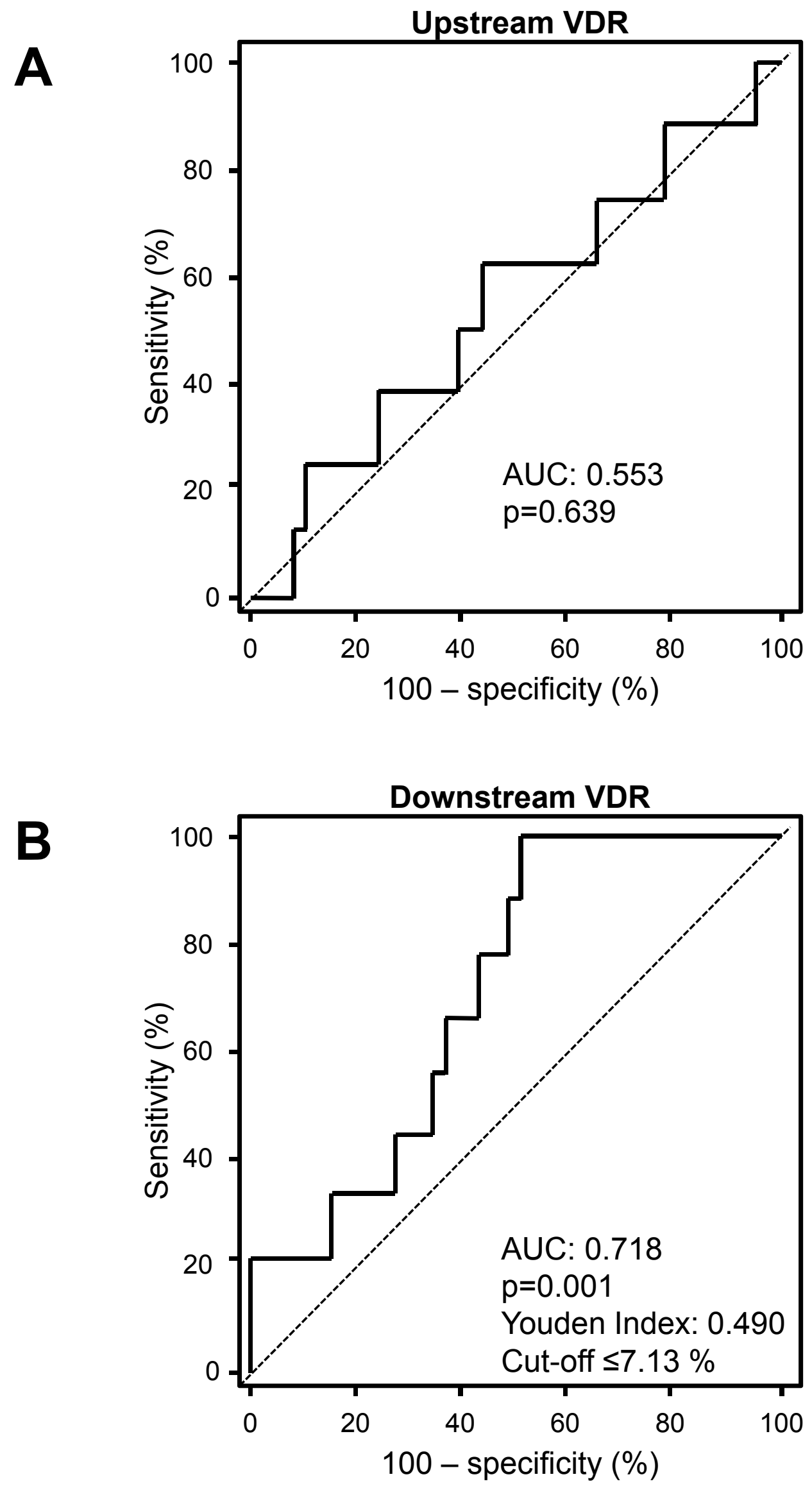


\section{Figure 4}

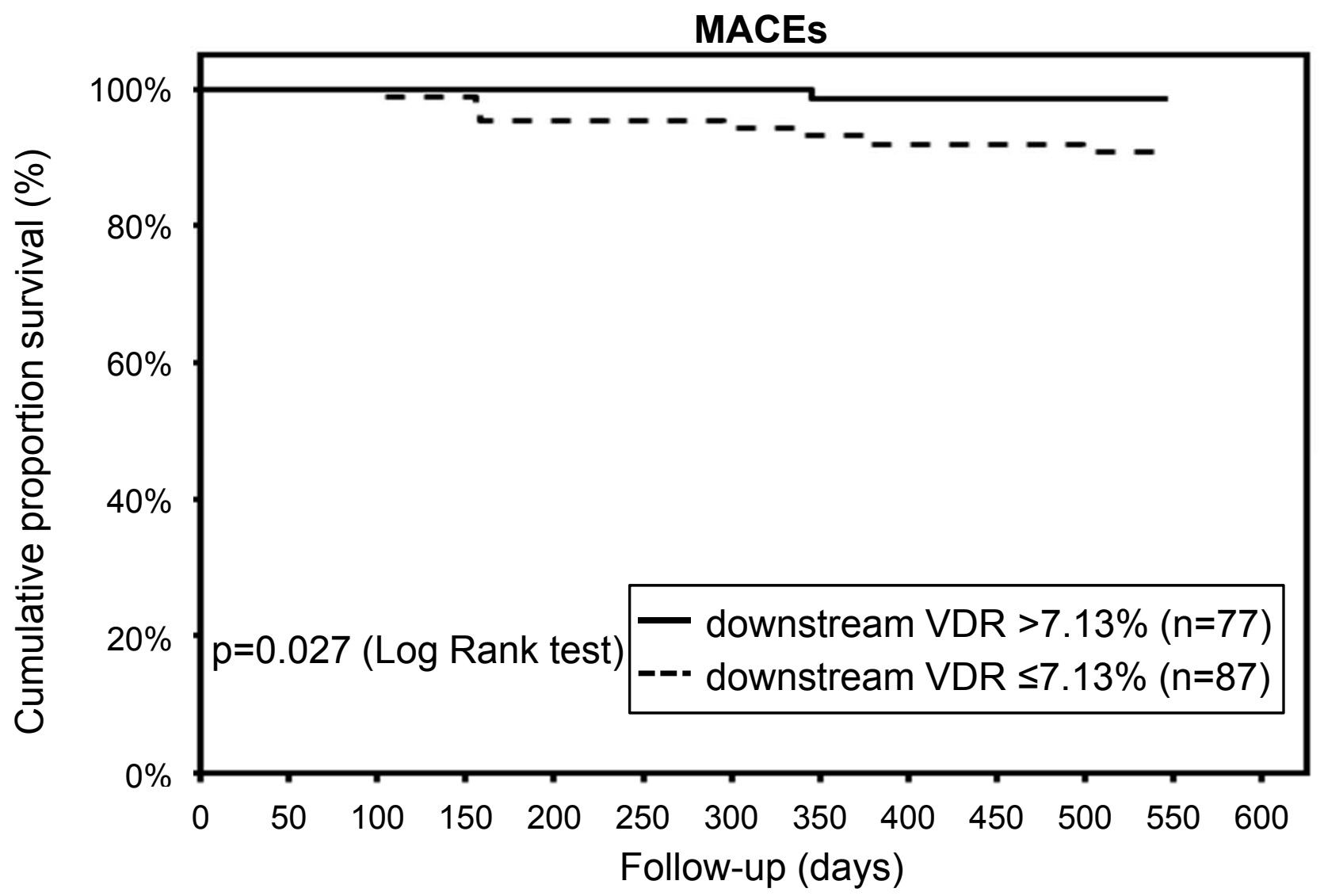




\section{Figure 5}
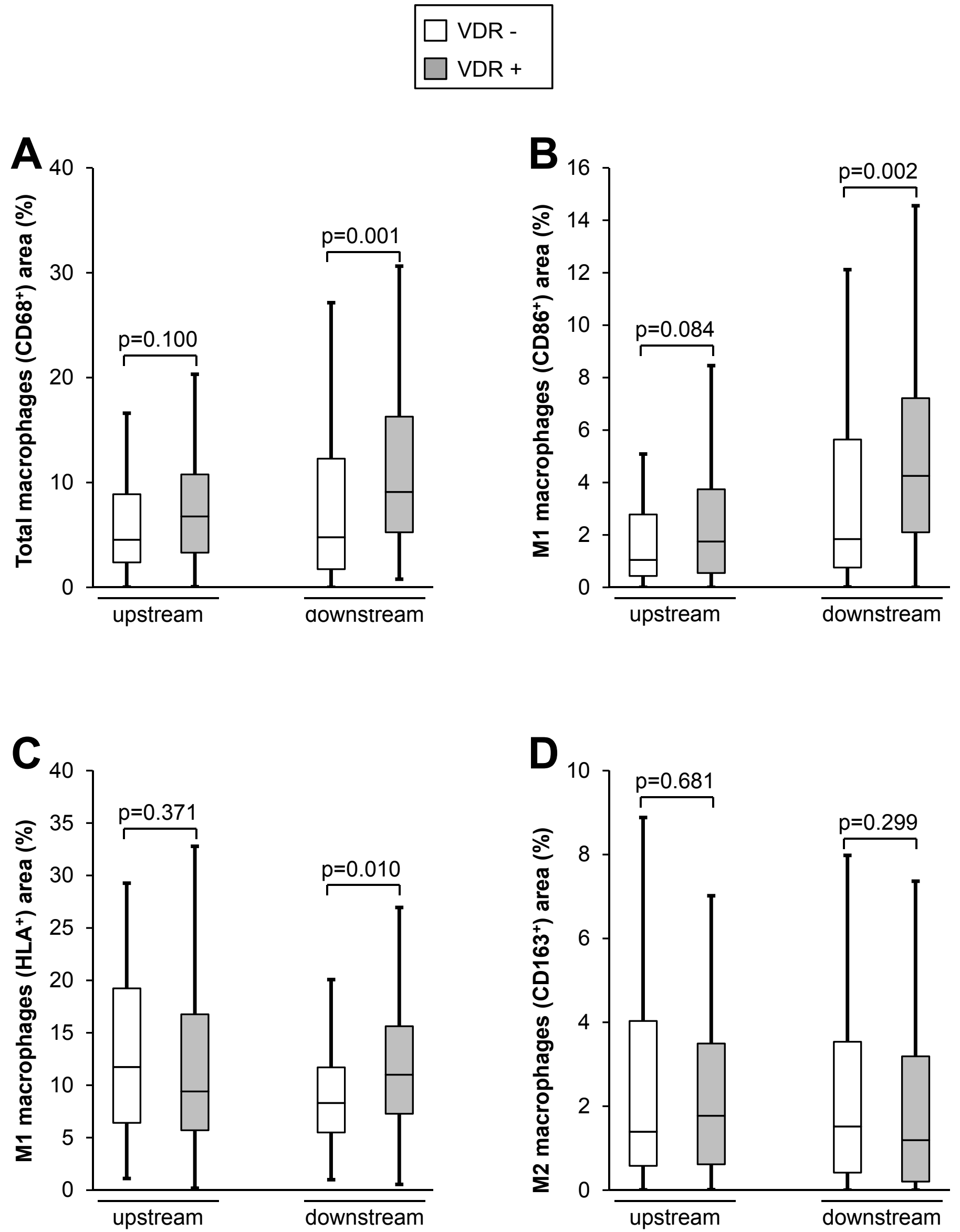


\section{Figure 6}
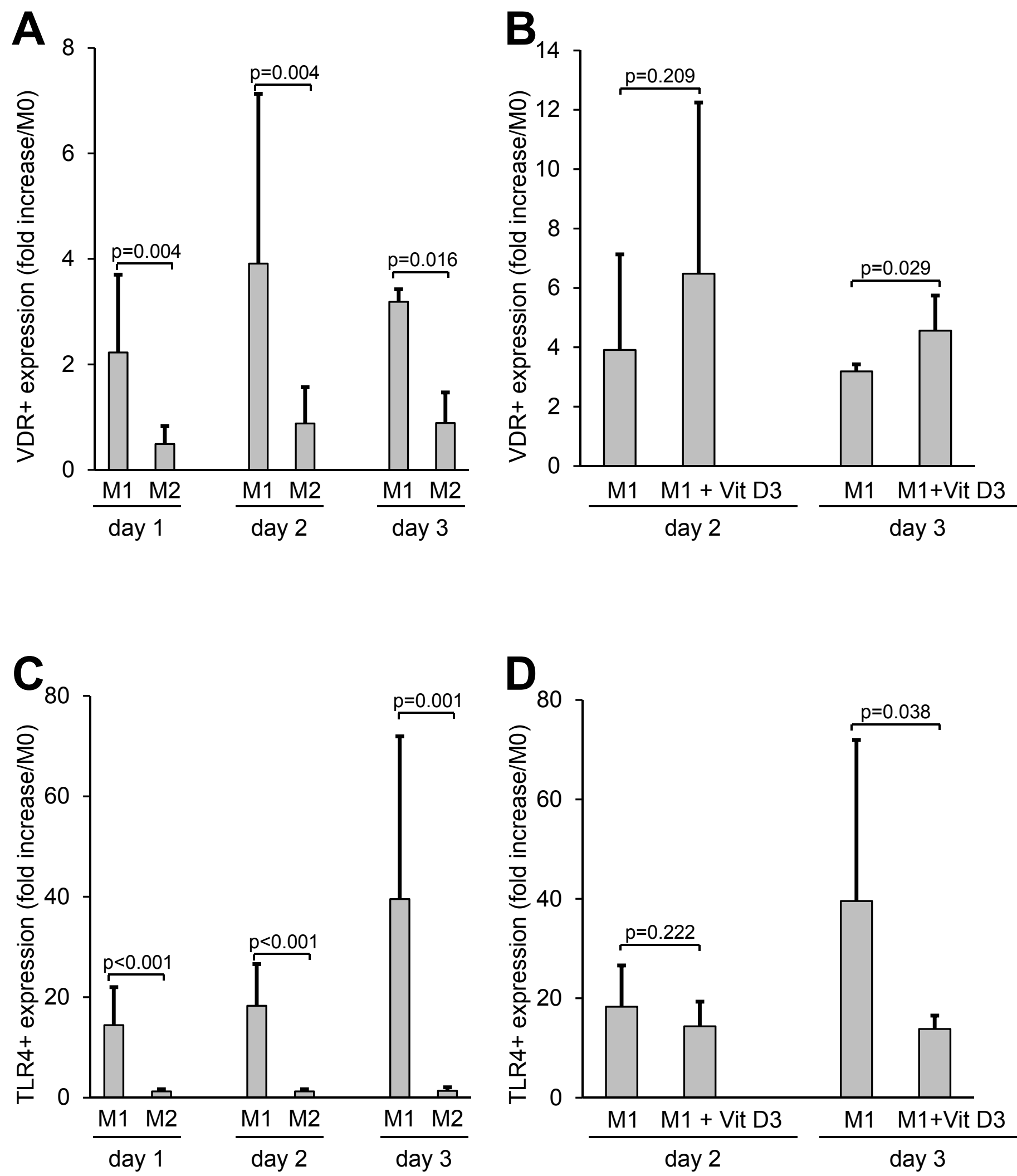
Table 1. Clinical characteristics of overall cohort at admission.

\begin{tabular}{|c|c|}
\hline & Overall cohort $(n=164)$ \\
\hline \multicolumn{2}{|l|}{ Demographic } \\
\hline Age, yr. (IQR) & $72(67-77)$ \\
\hline Males, no. $(\%)$ & $109(66.5)$ \\
\hline Symptomatic, no. (\%) & $34(20.73)$ \\
\hline Systolic BP*, mmHg (IQR) & $140(130-145)$ \\
\hline Diastolic BP, mmHg (IQR) & $80(80-90)$ \\
\hline Waist circumference $(\mathrm{cm})$ & $90(87-97)$ \\
\hline Carotid stenosis $(\%)$ & $80(75-90)$ \\
\hline Hypertension, no. (\%) & $118(71.9)$ \\
\hline Active smokers, no. $(\%)$ & $42(25.6)$ \\
\hline Previous smokers, no. (\%) & $73(44.5)$ \\
\hline Type 2 diabetes, no. (\%) & $31(18.3)$ \\
\hline Dyslipidaemia, no. $(\%)$ & $99(60.4)$ \\
\hline Chronic $\mathrm{CAD}^{\dagger}$, no. $(\%)$ & $28(17.1)$ \\
\hline \multicolumn{2}{|l|}{ Medications } \\
\hline RAAS $^{+}$inhibitors, no. (\%) & $86(49.4)$ \\
\hline ACE-I ${ }^{\S}$, no. $(\%)$ & $10(6.1)$ \\
\hline ARBs ${ }^{\|}$, no. $(\%)$ & $76(43.3)$ \\
\hline$\beta$-blockers, no. $(\%)$ & $48(29.3)$ \\
\hline Calcium antagonists, no. (\%) & $48(29.3)$ \\
\hline Diuretics, no. $(\%)$ & $19(11.6)$ \\
\hline Statins, no. (\%) & $86(52.4)$ \\
\hline Antiaggregants, no. (\%) & $132(80.4)$ \\
\hline Aspirin, no. $(\%)$ & $96(58.5)$ \\
\hline Thienopyridine, no. (\%) & $36(21.9)$ \\
\hline Anticoagulants (heparin) & $9(5.5)$ \\
\hline Oral antidiabetics, no. $(\%)$ & $23(14.0)$ \\
\hline Insulin, no. $(\%)$ & $4(2.4)$ \\
\hline \multicolumn{2}{|l|}{ Laboratory findings } \\
\hline \multicolumn{2}{|l|}{ Haematology } \\
\hline Total WBC ${ }^{\#}$, no. $\times 10^{9} / 1$ (IQR) & $7.23(6.26-8.35)$ \\
\hline Neutrophil, no. $\times 10^{9} / 1$ (IQR) & $4.64(3.65-5.53)$ \\
\hline Lymphocyte, no. x $10^{9} / 1$ (IQR) & $1.78(1.42-2.15)$ \\
\hline Monocyte, no. x 109/1 (IQR) & $0.45(0.37-0.57)$ \\
\hline Platelet, no. x $10^{9} / 1$ (IQR) & $232(192-280)$ \\
\hline Red blood cell, no. $\times 10^{12} / 1$ (IQR) & $4.70(4.40-4.95)$ \\
\hline \multicolumn{2}{|l|}{ Chemistry } \\
\hline Serum total-c ${ }^{* *} \mathrm{mg} / \mathrm{dl}(\mathrm{IQR})$ & $194(164-219)$ \\
\hline Serum LDL-c ${ }^{\dagger \dagger} \mathrm{mg} / \mathrm{dl}$ (IQR) & $116(89-143)$ \\
\hline Serum HDL-c ${ }^{*} \mathrm{mg} / \mathrm{dl}$ (IQR) & $49(42-61)$ \\
\hline Serum $\mathrm{TAG}^{\S \S} \mathrm{mg} / \mathrm{dl}$ (IQR) & $120(92-162)$ \\
\hline Fibrinogen, mg/dl (IQR) & $3.69(3.13-4.21)$ \\
\hline Fasting glycaemia, mg/dl (IQR) & $101(91-115)$ \\
\hline
\end{tabular}


Data are expressed as median (interquartile range [IQR]) or number [no.] (percentages [\%]).

* BP: blood pressure

$\uparrow$ CAD: coronary artery disease

† RAAS: renin-angiotensin-aldosterone system.

$\S$ ACE-I: angiotensin converting enzyme inhibitor

IIARBs: angiotensin receptor blockers

\# WBC: white blood cells

** total-c: total cholesterol

$\dagger \dagger$ LDL: low-density lipoprotein

t+ HDL: high-density lipoprotein

$\S \S \mathrm{TAG}$ : triglyceride 
Table 2. Serum levels of vitamin D, hs-CRP and CC chemokines.

\begin{tabular}{lc}
\hline & Overall cohort $(\mathbf{n}=\mathbf{1 6 4})$ \\
\hline $25(\mathrm{OH}) \mathrm{D}_{3}{ }^{*}, \mathrm{nmol} / \mathrm{l}(\mathrm{IQR})$ & $32.03(21.31-50.40)$ \\
\hline $\mathrm{CRP}^{\dagger}, \mu \mathrm{g} / \mathrm{ml}(\mathrm{IQR})$ & $2.34(0.87-4.99)$ \\
$\mathrm{CCL} 2^{*}, \mathrm{pg} / \mathrm{ml}(\mathrm{IQR})$ & $65.29(15.63-148.05)$ \\
$\mathrm{CCL} 3, \mathrm{pg} / \mathrm{ml}(\mathrm{IQR})$ & $8.24(7.80-12.18)$ \\
$\mathrm{CCL} 4, \mathrm{pg} / \mathrm{ml}(\mathrm{IQR})$ & $29.47(15.62-47.32)$ \\
$\mathrm{CCL} 5, \mathrm{ng} / \mathrm{ml}(\mathrm{IQR})$ & $54.23(33.3-91.01)$ \\
\hline
\end{tabular}

Data are expressed as median (interquartile range [IQR]).

* 25(OH) $\mathrm{D}_{3}$ : 25-hydroxyvitamin $\mathrm{D}$

$\dagger$ hsCRP: C-reactive protein

† CCL: chemokine (C-C motif) ligand 
Table 3. Relationship between serum levels of 25-hydroxyvitamin $D$ and intraplaque biomarkers.

\begin{tabular}{|c|c|c|}
\hline & \multicolumn{2}{|c|}{$25(\mathrm{OH}) \mathrm{D}_{3}{ }^{*}, \mathrm{nmol} / \mathrm{l}$} \\
\hline & $r$ & $p$-value \\
\hline \multicolumn{3}{|l|}{ Upstream portion } \\
\hline $\mathrm{VDR}^{\dagger}, \%$ & -0.034 & 0.685 \\
\hline Oil Red O, \% & 0.242 & 0.003 \\
\hline Total collagen, $\%$ & 0.044 & 0.594 \\
\hline Total macrophages, \% & 0.141 & 0.086 \\
\hline M1 subset $\left(\mathrm{CD} 86^{+}\right)^{\|}, \%$ & -0.008 & 0.919 \\
\hline M1 subset $\left(\mathrm{HLA}^{+}\right)^{\S}, \%$ & 0.101 & 0.233 \\
\hline M2 subset $\left(\mathrm{CD} 163^{+}\right), \%$ & -0.021 & 0.805 \\
\hline \multicolumn{3}{|l|}{ Downstream portion } \\
\hline VDR, \% & 0.166 & 0.038 \\
\hline Oil Red O, \% & 0.010 & 0.900 \\
\hline Total collagen, $\%$ & 0.106 & 0.190 \\
\hline Total macrophages, \% & 0.177 & 0.027 \\
\hline M1 subset $\left(\mathrm{CD} 86^{+}\right), \%$ & 0.083 & 0.308 \\
\hline M1 subset $\left(\mathrm{HLA}^{+}\right), \%$ & -0.133 & 0.101 \\
\hline $\mathrm{M} 2$ subset $\left(\mathrm{CD} 163^{+}\right), \%$ & -0.030 & 0.709 \\
\hline
\end{tabular}

Correlations were performed by Spearman's rank correlation coefficient.

* 25(OH) $\mathrm{D}_{3}$ : 25-hydroxyvitamin D

†VDR: vitamin D receptor

IC CD: cluster of differentiation

$\S$ HLA: human leukocyte antigen 
Table 4. Correlation between vitamin $D$ receptor expression and intraplaque biomarkers.

\begin{tabular}{lcc}
\hline \multicolumn{3}{c}{ Upstream } \\
\hline VDR $^{*}, \%$ vs. & $r$ & $p$-value \\
\cline { 2 - 3 } Oil Red O, \% & 0.016 & 0.621 \\
Total collagen, \% & -0.105 & 0.482 \\
Total macrophages, \% & 0.065 & 0.408 \\
M1 subset (CD86 $\left.{ }^{+}\right)^{\dagger}, \%$ & 0.371 & $<\mathbf{0 . 0 0 1}$ \\
M1 subset $\left(\mathrm{HLA}^{+}\right)^{\|}, \%$ & 0.402 & $<\mathbf{0 . 0 0 1}$ \\
M2 subset $\left(\mathrm{CD} 163^{+}\right), \%$ & 0.148 & 0.072 \\
\hline
\end{tabular}

\begin{tabular}{lcc}
\hline \multicolumn{3}{c}{ Downstream } \\
\hline VDR, \% vs. & $r$ & $p$-value \\
\cline { 2 - 3 } Oil Red O, \% & -0.010 & 0.899 \\
Total collagen, \% & 0.048 & 0.541 \\
Total macrophages, \% & 0.365 & $<\mathbf{0 . 0 0 1}$ \\
M1 subset (CD86 ${ }^{+}$), \% & 0.279 & $<\mathbf{0 . 0 0 1}$ \\
M1 subset (HLA $\left.{ }^{+}\right), \%$ & 0.304 & $<\mathbf{0 . 0 0 1}$ \\
M2 subset $\left(\mathrm{CD}^{+} 63^{+}\right), \%$ & -0.005 & 0.947 \\
\hline
\end{tabular}

Correlations were performed by Spearman's rank correlation coefficient.

* VDR: vitamin D receptor

$\uparrow \mathrm{CD}$ : cluster of differentiation

|| HLA: human leukocyte antigen 
Table 5. Cox hazard proportional model showing the predictive value of categorized downstream VDR expression (cutoff $\leq 7.13 \%$ ) towards MACE occurrence during 18 months of follow-up.

\begin{tabular}{lcccccccc}
\hline & \multicolumn{3}{c}{ Univariate model } & & \multicolumn{3}{c}{ Multivariate model } \\
& HR $^{*}$ & $95 \% \mathrm{CI}^{\dagger}$ & $p$-value & & HR & $95 \%$ CI & $p$-value \\
\hline MACEs $^{\dagger}$ & & & & & & & & \\
Downstream VDR & & 0.82 & $0.67-0.99$ & $\mathbf{0 . 0 4 6}$ & & 0.78 & $0.62-0.98$ & $\mathbf{0 . 0 3 2}$ \\
Age & 0.96 & $0.89-1.04$ & 0.382 & & 0.95 & $0.87-1.04$ & 0.271 \\
Gender, male & 0.97 & $0.24-3.88$ & 0.966 & & 0.61 & $0.14-2.68$ & 0.514 \\
Serum vitamin D & 1.01 & $0.98-1.02$ & 0.859 & & 1.01 & $0.98-1.02$ & 0.628 \\
\hline
\end{tabular}

* HR: hazards ratio

$\dagger$ CI: confidence interval

† MACEs: major adverse cardiovascular events

|| VDR: vitamin D receptor 